\title{
A Novel Bayesian Method for Fitting a Circle to Noisy Points
}

\author{
Marcus Baum,Vesa Klumpp, and Uwe D. Hanebeck \\ Intelligent Sensor-Actuator-Systems Laboratory (ISAS), \\ Institute for Anthropomatics, \\ Karlsruhe Institute of Technology (KIT), Germany. \\ \{marcus.baum, vesa.klumpp\}@kit.edu, uwe.hanebeck@ieee.org
}

\begin{abstract}
This paper introduces a novel recursive Bayesian estimator for the center and radius of a circle based on noisy points. Each given point is assumed to be a noisy measurement of an unknown true point on the circle that is corrupted with known isotropic Gaussian noise. In contrast to existing approaches, the novel method does not make assumptions about the true points on the circle, where the measurements stem from. Closed-form expressions for the measurement update step are derived. Simulations show that the novel method outperforms standard Bayesian approaches for circle fitting.
\end{abstract}

Keywords: Circle fitting, error-in-variables (EIV) model, nonlinear regression, Bayesian state estimation, tracking.

\section{Introduction}

Fitting a circle to noisy data points is a basic problem that arises frequently in many application areas. For instance, circular shapes have to be extracted from image data [1] in computer vision and pattern recognition. In mobile robotics, circle fitting algorithms are used for localizing circle-shaped landmarks [2, 3]. In target tracking applications, the spatial extent of a target can be modeled with a circular disc, which can be tracked with circle fitting algorithms [4]. Further application areas can be found, e.g., in physics, medicine, meteorology, and microwave measurement.

All these different applications impose different requirements and assumptions on the circle fitting algorithm [5]. For instance, in image processing applications the measurement noise is typically small and a large number of measurements is available. In tracking applications, however, one has to deal with high measurement noise and only a few measurements. Sometimes data points are given from the entire circle, and sometimes the data points only lie on a small arc. In several scenarios, the circle fit must be very accurate while the computation time is less important. On the other hand, for real-time applications, highly efficient and fast algorithms are necessary. These different prerequisites have led to many different algorithms and methodologies for fitting circles.

In this paper, we focus on tracking applications in which the measurements are received sequentially and the circle may change its position and radius over time. Each received measurement is assumed to stem from an unknown true point on the circle and is corrupted by stochastic noise. The measurement noise is a priori known as it results from a particular sensor such as a laser scanner.

We seek a Bayesian estimator for the parameters of the circle. A Bayesian state estimator is a recursive update scheme for a probability distribution over the unknown state according to Bayes' rule [6]. The temporal evolution of the state is captured with a stochastic system model, which allows to propagate the uncertainty about the state to the next time step.

The major challenge in circle fitting is that the true points, where the measurements stem from, are unknown. Existing Bayesian methods all make assumptions on the locations of the true points on the circle. Naturally, when these assumptions are not justified in an application, poor estimation results may be obtained. This paper introduces a novel Bayesian method for estimating the parameters of a circle. In contrast to existing approaches, no assumptions on the location of true points on the circle are made, i.e., no distinctive probability distribution is defined. Closed-form expressions for the measurement update step are derived. The obtained method yields more precise estimation results than traditional Bayesian approaches, especially in scenarios with high measurement noise.

\section{Problem Formulation}

We consider the problem of estimating the parameters of a circle based on noisy measurements of (unknown) true points lying on the circle.

For the sake of simplicity, we assume that the cir- 
cle is static and does not change its parameters over time. An extension of the new method to the dynamic case is straightforward and can be done by propagating the resulting probability densities through a stochastic motion model.

The given two-dimensional points $\underline{\hat{z}}_{k}=\left[\hat{x}_{k}, \hat{y}_{k}\right]^{T}$ with time index $k$ are measurements of true unknown points $\underline{\tilde{z}}_{k}=\left[\tilde{x}_{k}, \tilde{y}_{k}\right]^{T}$ on the circle according to

$$
\underline{\hat{z}}_{k}=\underline{\tilde{z}}_{k}+\underline{\epsilon}_{k}
$$

where $\underline{\epsilon}_{k}{ }^{1}$ is zero-mean isotropic Gaussian noise with known covariance matrix $\operatorname{diag}\left(\left[\sigma^{2}, \sigma^{2}\right]\right)$. The true points $\underline{\tilde{z}}_{k}$ are known to fulfill the implicit functional relationship

$$
g\left(\underline{\tilde{z}}_{k}, \underline{\tilde{p}}\right)=\left(\tilde{x}_{k}-\tilde{a}\right)^{2}+\left(\tilde{y}_{k}-\tilde{b}\right)^{2}-\tilde{r}^{2}=0,
$$

with the parameter vector $\underline{\tilde{p}}=[\tilde{a}, \tilde{b}, \tilde{r}]^{T}$ of the true circle and the circular shape function $g(\cdot, \cdot)$. The goal is to estimate the parameter vector $\underline{\tilde{p}}$ with a recursive Bayesian estimator. For this purpose, the uncertainty about the parameter vector $\tilde{p}$ having received measurements $\underline{\hat{z}}_{1}, \ldots, \underline{\hat{z}}_{k-1}$ is modeled with a random vector $\underline{\boldsymbol{p}}_{k-1}=\left[\boldsymbol{a}_{k-1}, \boldsymbol{b}_{k-1}, \boldsymbol{r}_{k-1}\right]^{T}$. By incorporating the next measurement $\underline{\underline{z}}_{k}$ according to Bayes' rule, the probability density of the updated estimate $\underline{\boldsymbol{p}}_{k}$ is computed.

The above specified circle fitting problem cannot be directly formulated as a Bayesian state estimation problem. The reason is that no conditional density $f\left(\underline{\tilde{z}}_{k} \mid \underline{p}_{k-1}\right)$, which determines the distribution of the true points on the circle, is available. Hence, the likelihood $f\left(\underline{\hat{z}}_{k} \mid \underline{p}_{k-1}\right)$ cannot be stated directly. It is important to note that this problem can be considered as a so-called errors-in-variables (EIV) model [5, 7], which is fundamentally different from the classical regression problem since both the independent and dependent variable are corrupted by stochastic noise.

The contribution of this paper is to derive a novel Bayesian state estimator for the circle fitting problem that does not make use of a conditional density function $f\left(\underline{\tilde{z}}_{k} \mid \underline{p}_{k-1}\right)$. The major insight is that it is actually not necessary to make any assumptions on the true points.

\section{Related Work}

This section outlines current methods for fitting circles to noisy data, where the focus lies on Bayesian methods. A recent overview of circle fitting algorithms can be found in [5].

\subsection{Bayesian Approaches}

One approach for constructing a Bayesian estimator for the circle fitting problem is to assume that the true points on the circle are drawn from a particular

\footnotetext{
${ }^{1}$ Note that all random variables are printed bold face in this paper.
}

(known) probability distribution $f\left(\underline{\tilde{z}}_{k} \mid \underline{p}_{k-1}\right)$. Then, a Bayesian estimator can be derived, because $f\left(\underline{\hat{z}}_{k} \mid \underline{p}_{k-1}\right)$ is determined [8]. The problem with this approach is that no closed-form expressions for the measurement update can be derived. Furthermore, in many applications there is no justification for choosing a specific density $f\left(\underline{\tilde{z}}_{k} \mid \underline{p}_{k-1}\right)$. For instance, if we choose a uniform distribution on the circle for the true points, the estimator yields bad results if the true points lie in fact on a circular arc. A remedy for this problem is to estimate the conditional density $f\left(\underline{\tilde{z}}_{k} \mid \underline{p}_{k-1}\right)$ together with the parameters $\tilde{p}$. However, this solution comes with other disadvantages. First, the number of unknown parameters to be estimated increases dramatically and typically no closed-form solutions are available such that Monte Carlo methods have to be employed. Second, already the assumption that the true points are drawn from a probability density may be too restrictive.

An alternative solution is to linearize the constraint (1) around the measurement $\underline{\hat{z}}_{k}$ and the current estimate of the circle parameters $[9,10,11]$ in order to obtain a linear measurement equation in the form $\underline{\boldsymbol{z}}_{k}^{*}=\mathbf{H}_{k} \underline{\boldsymbol{p}}_{k-1}+\boldsymbol{w}_{k}^{*}$, where $\underline{\boldsymbol{z}}_{k}^{*}$ and $\boldsymbol{w}_{k}^{*}$ result from the linearization. It is important to note that in contrast to the standard Extended Kalman Filter, it is also necessary to linearize around the current measurement. Hence, it is implicitly assumed that the measurement coincides with the true point. As a consequence, the linearization error is not only high if the current parameter estimate is bad, but also when the measurement is far away from the true point on the circle. Unfortunately, such cases often occur in tracking applications, e.g., when the measurement noise or the system noise is high. Furthermore, due to the linearization, it is not possible to perform gating, which is in particular necessary for tracking multiple circles.

In [12], a Bayesian method based on analyzing the residual gradient direction was proposed. However, since the gradient direction is nonlinear with respect to the true points, it is also necessary to linearize around the current measurement.

\subsection{Non-Bayesian Approaches}

A popular method for fitting circles is orthogonal distance regression [5, 13], which aims at minimizing the cost function $\mathcal{F}_{2}=\sum d_{k}^{2}$, where $d_{k}=$ $\sqrt{\left(x_{k}-a\right)^{2}+\left(y_{k}-b\right)^{2}}-r$ is the orthogonal geometric distance from the measurement to the circle. Since this is a nonlinear least-squares problem for which no closed-form solutions can be derived, iterative optimization methods, like the Gauss-Newton method or the Levenberg-Marquardt algorithm [5] have to be used to find an approximate solution.

An alternative to the geometric fit is the so-called algebraic fit, which minimizes $\mathcal{F}_{2}=\sum f_{k}^{2}$ where $f_{i}$ is a simple algebraic formula. For instance, the widely-used 
Delogne-Kåsa method [5] works with $f_{k}=\left(x_{k}-a\right)^{2}+$ $\left(y_{k}-b\right)^{2}-r^{2}$. A change of parameter in this cost functions yields a linear least-squares problem, which can be solved recursively in closed form. In general, geometric fits are more accurate than algebraic fits. However, geometric fits are computationally far more expensive and may lead to local minima. The geometric and algebraic fit do not make use of a statistical description of the problem as in Section 2. Hence, they do not directly allow to incorporate information about the measurement noise.

Statistical formulations of the circle fitting problem can be found in $[5,14,15,16]$. In the functional model, the true points are assumed to be fixed but unobservable, it is only known that they lie on the circle. In this case, the true points are treated as additional parameters of the model. In the structural model, the true points are assumed to be independent realizations of a random variable with a probability distribution concentrated on the circle. As pointed out in [15], the Maximum Likelihood Estimator (MLE) coincides with the geometric fit in case of the functional model. However, the MLE in the structural model is different from the geometric fit. Based on the statistical models, a statistical error analysis of circle fitting algorithms can be performed $[5,14]$. This analysis includes bias and variance estimation of the circle fits.

\section{Novel Approach}

Let us assume that the circle is centered at the position $[\tilde{a}, \tilde{b}]^{T}$ with radius $\tilde{r}$. Now, we consider the sequence of random variables

$$
\boldsymbol{d}_{k}=\left\|\underline{\boldsymbol{z}}_{k}-[\tilde{a}, \tilde{b}]^{T}\right\|
$$

where the random variable $\boldsymbol{d}_{k}$ is the distance from the random variable $\underline{\boldsymbol{z}}_{k}=\underline{\tilde{z}}_{k}+\underline{\boldsymbol{\epsilon}}_{k}$ to the center $[\tilde{a}, \tilde{b}]^{T}$. Note that the measurement $\underline{\hat{z}}_{k}$ is an observation of the random variable $\underline{\boldsymbol{z}}_{k}$.

Due to the assumption of isotropic Gaussian noise, the statistics of $\boldsymbol{d}_{k}$ does only depend on the radius $\tilde{r}$ and variance $\sigma^{2}$. It actually does not matter, where the true point $\underline{\tilde{z}}_{k}$ lies on the circle. A visualization of this property is given in Fig. 1. Due to the rotational invariance of the circle and the noise, the location of the true point $\underline{\tilde{z}}_{k}$ on the true circle does not influence the random distance of the measurement to the center. Actually, this property allows us to estimate $\underline{\tilde{p}}$ without explicitly estimating $\underline{\tilde{z}}_{k}$. For this purpose, we will first formalize these insights. We make use of the squared Euclidean distance in order to obtain simpler expressions. For given parameters $[\tilde{a}, \tilde{b}, \tilde{r}]^{T}$, we obtain

$$
\begin{aligned}
\boldsymbol{d}_{k}^{2} & =\left(\boldsymbol{x}_{k}-\tilde{a}\right)^{2}+\left(\boldsymbol{y}_{k}-\tilde{b}\right)^{2} \\
& =\left(\tilde{x}_{k}-\tilde{a}\right)^{2}+\left(\tilde{y}_{k}-\tilde{b}\right)^{2}+\boldsymbol{w}_{k} \\
& =\tilde{r}^{2}+\boldsymbol{w}_{k},
\end{aligned}
$$

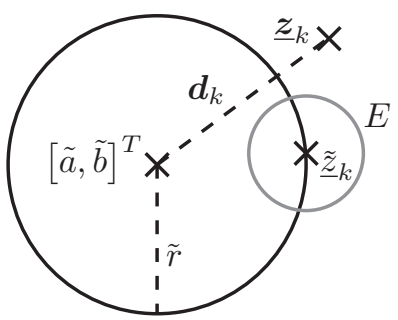

Figure 1: The distance $\boldsymbol{d}_{k}$ from the center $[\tilde{a}, \tilde{b}]^{T}$ to the observed random vector $\underline{\boldsymbol{z}}_{k}=\underline{\tilde{z}}_{k}+\underline{\boldsymbol{\epsilon}}_{k}$ does not depend on the true point $\underline{\tilde{z}}_{k}$, since the covariance ellipse $E$ is isotropic, i.e., a circle.

where $\boldsymbol{w}_{k}$ is a proper noise term whose statistics depends only on $\tilde{r}^{2}$ and $\sigma^{2}$.

Theorem 1. The statistics of $\boldsymbol{w}_{k}$ only depend on $\tilde{r}^{2}$ and the variance $\sigma^{2}$ of the measurement noise. Its first two moments are given by $\mathrm{E}\left\{\boldsymbol{w}_{k}\right\}=2 \sigma^{2}$ and $\operatorname{Var}\left\{\boldsymbol{w}_{k}\right\}=4\left(\sigma^{4}+\sigma^{2} \tilde{r}^{2}\right)$.

Proof. With the abbreviation $\overline{\boldsymbol{a}}_{k}:=\boldsymbol{x}_{k}-\tilde{a}$ and $\overline{\boldsymbol{b}}_{k}:=$ $\boldsymbol{y}_{k}-\tilde{b}$, we have $\overline{\boldsymbol{a}}_{k} \sim \mathcal{N}\left(\tilde{x}_{k}-\tilde{a}, \sigma^{2}\right)$ and $\overline{\boldsymbol{b}}_{k} \sim \mathcal{N}\left(\tilde{y}_{k}-\right.$ $\left.\tilde{b}, \sigma^{2}\right)$. Then,

$$
\frac{1}{\sigma^{2}} \boldsymbol{d}_{k}^{2}=\frac{1}{\sigma^{2}}\left(\overline{\boldsymbol{a}}_{k}^{2}+\overline{\boldsymbol{b}}_{k}^{2}\right) \sim \text { noncentral- } \chi^{2}(k, \lambda),
$$

where $k=2$ and $\lambda=\frac{1}{\sigma^{2}}\left(\left(\tilde{x}_{k}-\tilde{a}\right)^{2}+\left(\tilde{y}_{k}-\tilde{b}\right)^{2}\right)=\frac{1}{\sigma^{2}} \tilde{r}^{2}$ only depend on $\tilde{r}^{2}$ and $\sigma$. The first two moments of $\frac{1}{\sigma^{2}} \boldsymbol{d}_{k}^{2}$ are

- $\mathrm{E}\left\{\frac{1}{\sigma^{2}} \boldsymbol{d}_{k}^{2}\right\}=2+\lambda=2+\frac{1}{\sigma^{2}} \tilde{r}^{2}$, and

- $\operatorname{Var}\left\{\frac{1}{\sigma^{2}} \boldsymbol{d}_{k}^{2}\right\}=4+4 \cdot \lambda=4+\frac{4}{\sigma^{2}} \tilde{r}^{2}$.

As a consequence, the first two moments of $\boldsymbol{w}_{k}$ are $\mathrm{E}\left\{\boldsymbol{w}_{k}\right\}=2 \sigma^{2}$ and $\operatorname{Var}\left\{\boldsymbol{w}_{k}\right\}=4\left(\sigma^{4}+\sigma^{2} \tilde{r}^{2}\right)$.

Remark 1. Equation (2) can also be expressed in terms of the circular shape function according to

$$
g\left(\underline{\tilde{p}}, \underline{\boldsymbol{z}}_{k}\right)=\boldsymbol{w}_{k} .
$$

In order to construct a recursive Bayesian estimator for $\underline{\tilde{p}}$, we assume that the random vector $\underline{\boldsymbol{p}}_{k-1}=$ $\left[\boldsymbol{a}_{k-1}, \boldsymbol{b}_{k-1}, \boldsymbol{r}_{k-1}\right]^{T} \sim \mathcal{N}\left(\underline{\mu}_{k-1}^{p}, \mathbf{C}_{k-1}\right)$ models the uncertainty about $\underline{\tilde{p}}$ given the measurements $\underline{\hat{z}}_{1}, \ldots, \underline{\underline{\hat{z}}}_{k-1}$. The updated estimate with the next measurement $\underline{\hat{z}}_{k}$ is then

$$
\underline{\boldsymbol{p}}_{k} \sim f\left(\underline{p}_{k-1} \mid g\left(\underline{\boldsymbol{p}}_{k-1}, \underline{\hat{z}}_{k}\right)=\boldsymbol{w}_{k}\right),
$$

because it is known that the true parameter vector $\underline{\tilde{p}}$ satisfies the constraint $g\left(\underline{\tilde{p}}, \underline{\hat{z}}_{k}\right)=\boldsymbol{w}_{k}$. If we define the likelihood function

$$
f^{L}\left(\underline{\hat{z}}_{k} \mid \underline{p}_{k-1}\right):=f_{w_{k}}\left(g\left(\underline{p}_{k-1}, \underline{\hat{z}}_{k}\right)\right),
$$

where $f_{w_{k}}(\cdot)$ is the probability density of $\boldsymbol{w}_{k}$, the updated estimate can also be written as

$$
\underline{\boldsymbol{p}}_{k} \sim c_{k} \cdot f^{L}\left(\underline{\hat{z}}_{k} \mid \underline{p}_{k-1}\right) \cdot f\left(\underline{p}_{k-1}\right),
$$


where $c_{k}$ is normalization constant. Intuitively, the likelihood $f^{L}\left(\underline{\hat{z}}_{k} \mid \underline{p}_{k-1}\right)$ gives the probability that a circle with parameter vector $\underline{p}_{k-1}$ observes a measurement $\underline{\hat{z}}_{k}$ with quadratic distance $\left\|\underline{\hat{z}}_{k}-\left[a_{k-1}, b_{k-1}\right]^{T}\right\|^{2}$ to its center. This likelihood is in fact independent of the true point $\underline{\tilde{z}}_{k}$. It only depends on the variance $\sigma^{2}$ of the measurement noise and the true radius $\tilde{r}$ (see Theorem 1).

This fusion procedure can also be expressed by means of the following measurement equation

$$
g\left(\underline{\boldsymbol{p}}_{k-1}, \underline{\hat{z}}_{k}\right)-\boldsymbol{w}_{k}=0
$$

with a fictitious measurement 0 .

The noise term $\boldsymbol{w}_{k}$ is not Gaussian and it depends on the true unknown squared radius $\tilde{r}^{2}$. However, it can be approximated at each time step with a Gaussian distribution, which is (assumed to be) independent of $\underline{\boldsymbol{p}}_{k-1}$, by using the current estimate $\mathrm{E}\left\{\boldsymbol{r}_{k-1}^{2}\right\}$ for $\tilde{r}^{2}$, i.e.,

$$
\begin{aligned}
\mathrm{E}\left\{\boldsymbol{w}_{k}\right\} & =2 \sigma^{2} \\
\operatorname{Var}\left\{\boldsymbol{w}_{k}\right\} & \approx 4\left(\sigma^{4}+\sigma^{2} \mathrm{E}\left\{\boldsymbol{r}_{k-1}^{2}\right\}\right),
\end{aligned}
$$

where $\mathrm{E}\left\{\boldsymbol{r}_{k-1}^{2}\right\}=\mathrm{E}\left\{\boldsymbol{r}_{k-1}\right\}^{2}+\operatorname{Var}\left\{\boldsymbol{r}_{k-1}\right\}$.

In general, a measurement update according the measurement equation (3) could be performed with an arbitrary nonlinear Bayesian state estimator. However, since the measurement equation (3) only involves quadratic terms, exact analytic expressions for the first two moments of the updated estimate can be derived (see the following section).

It is interesting to note that with the change of parameters $\boldsymbol{A}_{k-1}=-2 \boldsymbol{a}_{k-1}, \boldsymbol{C}_{k-1}=-2 \boldsymbol{b}_{k-1}$, and $\boldsymbol{D}_{k-1}=\boldsymbol{a}_{k-1}^{2}+\boldsymbol{b}_{k-1}^{2}-\boldsymbol{r}_{k-1}^{2}$, the measurement equation (3) becomes linear [5]. However, this results in more complicated expressions for the statistics of $\boldsymbol{w}_{k}$. Simulations have shown that the estimation results are nearly the same for both types of parameters. However, in tracking applications one typically has to deal with dynamic systems, which are modeled with a motion equation in terms of the radius and center of the circle. A change of parameters would also require to rewrite the motion equation to a more complex expression. Thus, it is preferable to work directly with the center and radius.

If we choose the parameters $\boldsymbol{A}_{k-1}, \boldsymbol{C}_{k-1}$, and $\boldsymbol{D}_{k-1}$, the new method could be interpreted as a stochastic recursive version of the Delogne-Kasa method [5] enhanced with an adaptive unbiasing and reweighting procedure.

\section{Analytic Expressions for the Measurement Update Step}

The moments of a polynomially transformated Gaussian random variable can be calculated analytically [17].
The measurement equation (3) only involves quadratic expressions. Hence, it is possible to derive analytic expressions for the first two moments of the updated estimate $\underline{\boldsymbol{p}}_{k} \sim \mathcal{N}\left(\underline{\mu}_{k}^{p}, \mathbf{C}_{k}^{p}\right)$, which results from updating the previous estimate $\underline{\boldsymbol{p}}_{k-1} \sim \mathcal{N}\left(\underline{\mu}_{k-1}^{p}, \mathbf{C}_{k-1}^{p}\right)$ with the next measurement $\underline{\hat{z}}_{k}$. For this purpose, we need the following theorem, which gives analytic formulas for the noncentral moments of a multivariate Gaussian distribution [17].

Theorem 2. If $\left[\boldsymbol{z}_{1}, \ldots, \boldsymbol{z}_{n}\right]^{T} \sim \mathcal{N}(\mu, \Sigma)$ is a $n$ dimensional Gaussian distributed random vector with mean $\mu$ and covariance matrix $\Sigma$, then for nonnegative integers $s_{1}$ to $s_{n}$

$$
\begin{gathered}
\mathrm{E}\left\{\prod_{i=1}^{n} \boldsymbol{z}_{i}^{s_{i}}\right\}=\sum_{\nu_{1}=0}^{s_{1}} \ldots \sum_{\nu_{n}=0}^{s_{n}} \sum_{r=0}^{[s / 2]}(-1)^{\sum_{i=1}^{n} \nu_{i}} . \\
\left(\begin{array}{c}
s_{1} \\
\nu_{n}
\end{array}\right) \ldots\left(\begin{array}{l}
s_{n} \\
\nu_{n}
\end{array}\right) \frac{\left(\underline{h}^{T} \Sigma \underline{h}\right)^{r}\left(\underline{h}^{T} \mu\right)^{s-2 r}}{r !(s-2 r) !},
\end{gathered}
$$

where $s=s_{1}+\ldots+s_{n}$ and $\underline{h}=\left[\frac{s_{1}}{2}-\nu_{1}, \ldots, \frac{s_{n}}{2}-\nu_{n}\right]^{T}$.

The first step is to derive closed-form expressions for the first two moments of the random vector

$$
\left[\begin{array}{c}
\underline{\boldsymbol{p}}_{k-1} \\
\boldsymbol{h}_{k}
\end{array}\right]=\left[\begin{array}{c}
\boldsymbol{a}_{k-1} \\
\boldsymbol{b}_{k-1} \\
\boldsymbol{r}_{k-1} \\
\left(\hat{x}_{k}-\boldsymbol{a}_{k-1}\right)^{2}+\left(\hat{y}_{k}-\boldsymbol{b}_{k-1}\right)^{2}-\boldsymbol{r}_{k-1}^{2}-\boldsymbol{w}_{k}
\end{array}\right]
$$

For this purpose, we make use of the substitution $\overline{\boldsymbol{a}}_{k}:=$ $\hat{x}_{k}-\boldsymbol{a}_{k-1}$ and $\overline{\boldsymbol{b}}_{k}:=\hat{y}_{k}-\boldsymbol{b}_{k-1}$ with $\overline{\boldsymbol{a}}_{k} \sim \mathcal{N}\left(\hat{x}_{k}-\right.$ $\left.\mu_{k-1}^{a}, \sigma^{2}\right)$ and $\overline{\boldsymbol{b}}_{k} \sim \mathcal{N}\left(\hat{y}_{k}-\mu_{k-1}^{b}, \sigma^{2}\right)$. The mean is then given by

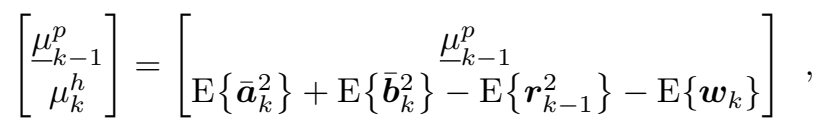

whose last component can be calculated with the help of Theorem 2. The covariance matrix of $\left[\underline{\boldsymbol{p}}_{k-1}, \boldsymbol{h}_{k}\right]^{T}$ is composed as follows

$$
\begin{aligned}
& \operatorname{Cov}\left\{\left[\begin{array}{c}
\underline{\boldsymbol{p}}_{k-1} \\
\boldsymbol{h}_{k}
\end{array}\right]\right\}= {\left[\begin{array}{cc}
\mathbf{C}_{k-1}^{p} & \mathbf{C}_{k}^{p, h} \\
\left(\mathbf{C}_{k}^{p, h}\right)^{T} & \mathbf{C}_{k}^{h}
\end{array}\right], \text { with } } \\
& \mathbf{C}_{k}^{p, h}=\left[\begin{array}{l}
\mathrm{E}\left\{\boldsymbol{a}_{k-1} \boldsymbol{h}_{k}\right\}-\mu_{k-1}^{a} \mu_{k}^{h} \\
\mathrm{E}\left\{\boldsymbol{b}_{k-1} \boldsymbol{h}_{k}\right\}-\mu_{k-1}^{b} \mu_{k}^{h} \\
\mathrm{E}\left\{\boldsymbol{r}_{k-1} \boldsymbol{h}_{k}\right\}-\mu_{k-1}^{r} \mu_{k}^{h}
\end{array}\right]
\end{aligned}
$$

and $\mathbf{C}_{k}^{h}=\mathrm{E}\left\{\left(\boldsymbol{h}_{k}\right)^{2}\right\}-\left(\mu_{k}^{h}\right)^{2}$. The components $\mathbf{C}_{k}^{h}$ and 
$\mathbf{C}_{k}^{p, h}$ can be computed with the identities

$$
\begin{aligned}
& \mathrm{E}\left\{\boldsymbol{a}_{k-1} \boldsymbol{h}_{k}\right\}=\mathrm{E}\left\{\left(\overline{\boldsymbol{a}}_{k}^{2}+\overline{\boldsymbol{b}}_{k}^{2}-\boldsymbol{r}_{k-1}^{2}+\boldsymbol{w}_{k}\right) \cdot\left(\overline{\boldsymbol{a}}_{k}+\hat{x}_{k}\right)\right\} \\
&=-\hat{x}_{k} \mathrm{E}\left\{\boldsymbol{r}_{k-1}^{2}\right\}-\hat{x}_{k} \mathrm{E}\left\{\boldsymbol{w}_{k}\right\}+\mathrm{E}\left\{\overline{\boldsymbol{a}}_{k} \overline{\boldsymbol{b}}_{k}^{2}\right\} \\
&-\mathrm{E}\left\{\boldsymbol{w}_{k}\right\} \mathrm{E}\left\{\overline{\boldsymbol{a}}_{k}\right\}+\hat{x}_{k} \mathrm{E}\left\{\overline{\boldsymbol{a}}_{k}^{2}\right\}+\mathrm{E}\left\{\overline{\boldsymbol{a}}_{k}^{3}\right\} \\
&+\hat{x}_{k} \mathrm{E}\left\{\overline{\boldsymbol{b}}_{k}^{2}\right\}-\mathrm{E}\left\{\boldsymbol{r}_{k-1}^{2}\right\} \mathrm{E}\left\{\overline{\boldsymbol{a}}_{k}\right\} \\
& \mathrm{E}\left\{\boldsymbol{b}_{k-1} \boldsymbol{h}_{k}\right\}=\mathrm{E}\left\{\left(\overline{\boldsymbol{b}}_{k}^{2}+\overline{\boldsymbol{a}}_{k}^{2}-\boldsymbol{r}_{k-1}^{2}+\boldsymbol{w}_{k+1}\right) \cdot\left(\overline{\boldsymbol{b}}_{k}+\hat{y}_{k}\right)\right\} \\
&=-\hat{y}_{k} \mathrm{E}\left\{\boldsymbol{r}_{k-1}^{2}\right\}-\hat{y}_{k} \mathrm{E}\left\{\boldsymbol{w}_{k}\right\}+\mathrm{E}\left\{\overline{\boldsymbol{b}}_{k} \overline{\boldsymbol{a}}_{k}^{2}\right\} \\
&-\mathrm{E}\left\{\boldsymbol{w}_{k}\right\} \mathrm{E}\left\{\overline{\boldsymbol{b}}_{k}\right\}+\hat{y}_{k} \mathrm{E}\left\{\overline{\boldsymbol{b}}_{k}^{2}\right\}+\mathrm{E}\left\{\overline{\boldsymbol{b}}_{k}^{3}\right\} \\
&+\hat{y}_{k} \mathrm{E}\left\{\overline{\boldsymbol{a}}_{k}^{2}\right\}-\mathrm{E}\left\{\boldsymbol{r}_{k-1}^{2}\right\} \mathrm{E}\left\{\overline{\boldsymbol{b}}_{k}\right\}, \\
& \mathrm{E}\left\{\boldsymbol{r}_{k-1} \boldsymbol{h}_{k}\right\}=\mathrm{E}\left\{\boldsymbol{r}_{k-1}^{2}\right\}-\mathrm{E}\left\{\boldsymbol{r}_{k-1}\right\} \mathrm{E}\left\{\boldsymbol{w}_{k}\right\} \\
&+\mathrm{E}\left\{\boldsymbol{r}_{k-1} \overline{\boldsymbol{a}}_{k}^{2}\right\}+\mathrm{E}\left\{\boldsymbol{r}_{k-1} \overline{\boldsymbol{b}}_{k}^{2}\right\}, \text { and } \\
& \mathrm{E}\left\{\left(\boldsymbol{h}_{k}\right)^{2}\right\}=\mathrm{E}\left\{\left(\overline{\boldsymbol{a}}_{k}^{2}+\overline{\boldsymbol{b}}_{k}^{2}-\boldsymbol{r}_{k-1}^{2}+\boldsymbol{w}_{k}\right)^{2}\right\} \\
&=\mathrm{E}\left\{\boldsymbol{r}_{k-1}^{4}\right\}+2 \mathrm{E}\left\{\boldsymbol{r}_{k-1}^{2} \boldsymbol{w}_{k}\right\}+\mathrm{E}\left\{\boldsymbol{w}_{k}^{2}\right\} \\
&-2 \mathrm{E}\left\{\boldsymbol{r}_{k-1}^{2} \overline{\boldsymbol{a}}_{k}^{2}\right\}-2 \mathrm{E}\left\{\boldsymbol{w}_{k}\right\} \mathrm{E}\left\{\overline{\boldsymbol{a}}_{k}^{2}\right\} \\
&-2 \mathrm{E}\left\{\boldsymbol{r}_{k-1}^{2} \overline{\boldsymbol{b}}_{k}^{2}\right\}-2 \mathrm{E}\left\{\boldsymbol{w}_{k}\right\} \mathrm{E}\left\{\overline{\boldsymbol{b}}_{k}^{2}\right\} \\
&+2 \mathrm{E}\left\{\overline{\boldsymbol{a}}_{k}^{2} \overline{\boldsymbol{b}}_{k}^{2}\right\}+\mathrm{E}\left\{\overline{\boldsymbol{b}}_{k}^{4}\right\}+\mathrm{E}\left\{\overline{\boldsymbol{a}}_{k}^{4}\right\}
\end{aligned}
$$

Due to the measurement equation (3), the updated estimate results from conditioning the probability density of $\left[\underline{\boldsymbol{p}}_{k-1}, \boldsymbol{h}_{k}\right]^{T}$ on $\boldsymbol{h}_{k}=0$. Assuming $\left[\underline{\boldsymbol{p}}_{k-1}, \boldsymbol{h}_{k}\right]^{T}$ to be Gaussian distributed, the updated estimate $\underline{\boldsymbol{p}}_{k} \sim$ $\mathcal{N}\left(\underline{\mu}_{k}^{p}, \mathbf{C}_{k}\right)$ becomes

$$
\begin{aligned}
\mu_{k}^{p} & =\underline{\mu}_{k-1}^{p}-\left(\mathbf{C}_{k}^{h}\right)^{-1} \mathbf{C}_{k}^{p, h} \mu_{k}^{h} \\
\mathbf{C}_{k}^{p} & =\mathbf{C}_{k-1}^{p}-\left(\mathbf{C}_{k}^{h}\right)^{-1}\left(\mathbf{C}_{k}^{p, h}\right)^{T} \mathbf{C}_{k}^{p, h},
\end{aligned}
$$

which results from the Kalman filter equation.

\section{Simulation}

This section shows typical examples with simulated data in which the novel method outperforms standard approaches for Bayesian circle fitting. The true circle is located at the position $[5,5]^{T}$ with radius 2. Furthermore, the prior information about the circle parameters is given by a Gaussian distribution with mean $[6,6,2.5]^{T}$ and covariance matrix $\operatorname{diag}(1,1,0.5)$.

First, we compare the new method with the Bayesian method suggested in [8], which assumes the unknown true points to be drawn from a uniform distribution on the circle. The uncertainty about the current estimate is captured with a Gaussian distribution. Since no closed-form expressions for the measurement update are available, we employ Monte Carlo sampling for approximating the posterior distribution. In this scenario, the true measured points are determined by sampling a Gaussian distribution with mean 0 and variance $\frac{1}{7}$, which restricts the angle of the true point to the $x$-axis. The variance of the isotropic measurement noise is given by $\sigma^{2}=0.2$. As shown in Fig. 2a and Fig. 2c, the novel method yields a much more better estimate for the true circle. The main reason for this is that the true points are not uniformly distributed on the circle as falsely assumed in [8]. The root-mean-square error (RMSE) of the parameter vector after the 20th measurement is 0.63 for the method suggested in [8] and 0.44 for the novel method (averaged over 100 runs). Hence, after the 20th measurement, the RMSE of the new method is about $30 \%$ smaller.

The second simulation compares the new method with the Extended Kalman Filter approach [9, 10, 11], which linearizes the circular shape function (1) at the measurement and the current estimate. The variance of the isotropic measurement noise is $\sigma^{2}=0.4$. Now the measurement sources are distributed uniformly on the true circle. Due to the high measurement noise, the linearization approach yields worse results, since the constraint is linearized at the measurement. However, the novel method provides an accurate circle estimate. The root-mean-square error of the parameter vector after the 20th measurement is 0.45 for the EKF approach and 0.37 for the novel method (averaged over 100 runs). One of these runs is visualized in Fig. 2b. The novel method is after the 20th measurement approximately $19 \%$ better than the EKF approach.

\section{Conclusions}

We have presented a novel Bayesian method for fitting a circle to noisy data. In contrast to existing methods, the novel method does not make assumptions on the true points on the circle, where the measurements stem from. The major theoretical insight is that it is actually not necessary to make any assumptions on the true points. We have shown typical examples in which the novel method outperforms standard methods. Future work consists of extending the approach to the case of unknown measurement noise. In this case, the measurement noise has to be estimated in addition to the circle parameters.

\section{References}

[1] I. Frosio and N. A. Borghese, "Real-time Accurate Circle Fitting with Occlusions," Pattern Recognition, vol. 41, no. 3, pp. 1041-1055, 2008.

[2] P. Nuez, R. Vazquez-Martin, A. Bandera, and F. Sandoval, "An Algorithm for Fitting 2-D Data on the Circle: Applications to Mobile Robotics," Signal Processing Letters, IEEE, vol. 15, pp. 127130, 2008.

[3] S. Zhang, L. Xie, and M. D. Adams, "Feature Extraction for Outdoor Mobile Robot Navigation based on a Modified Gauss-Newton Optimization Approach," Robotics and Autonomous Systems, vol. 54, no. 4, pp. $277-287,2006$. 


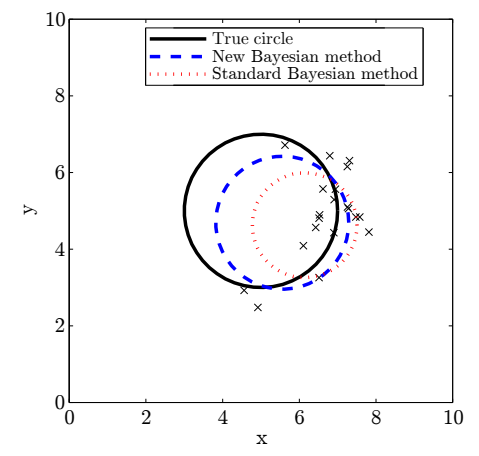

(a) Simulation 1: Comparison with the standard Bayesian approach.

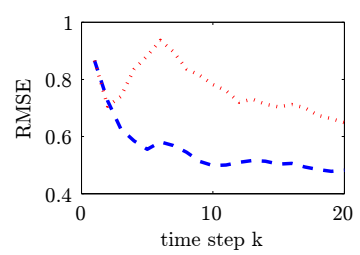

(c) RMSE for simulation 1 .

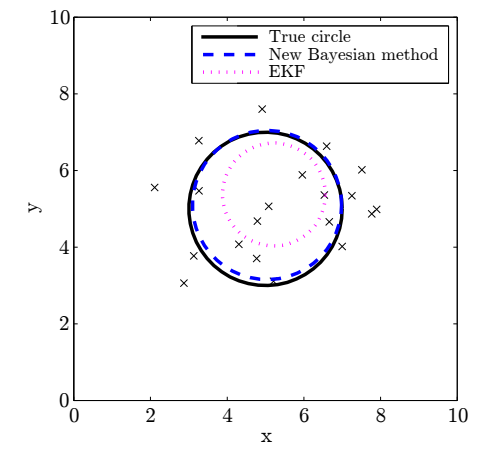

(b) Simulation 2: Comparison with the Extended Kalman Filter approach.

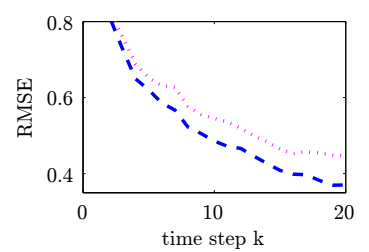

(d) RMSE for simulation 2 .

Figure 2: Example run for simulation 1 (a) and simulation 2 (b). RMSE for simulation 1 (c) and simulation 2 (d) averaged over 100 runs. The prior information about the circle parameters is given by a Gaussian distribution with mean $[6,6,2.5]$ and covariance matrix $\operatorname{diag}(1,1,0.5)$.

[4] M. Baum and U. D. Hanebeck, "Random Hypersurface Models for Extended Object Tracking," in Proceedings of the 9th IEEE International Symposium on Signal Processing and Information Technology (ISSPIT 2009), Ajman, United Arab Emirates, Dec. 2009.

[5] N. Chernov, Circular and Linear Regression: Fitting Circles and Lines by Least Squares. CRC Press, 2010.

[6] D. Hall and J. Linas, Handbook of Multisensor Data Fusion. $\quad$ CRC Press, May 2001.

[7] W. A. Fuller, Measurement Error Models. New York, NY, USA: John Wiley \& Sons, Inc., 1986.

[8] M. Werman and D. Keren, "A Bayesian Method for Fitting Parametric and Nonparametric Models to Noisy Data," IEEE Transactions on Pattern Analysis and Machine Intelligence, vol. 23, no. 5, pp. 528-534, 2001.

[9] J. Porrill, "Fitting Ellipses and Predicting Confidence Envelopes Using a Bias Corrected Kalman Filter," Image Vision Computing, vol. 8, pp. 3741, 1990.

[10] Z. Zhang, "Parameter Estimation Techniques: A Tutorial with Application to Conic Fitting," Image and Vision Computing, vol. 15, no. 1, pp. $59-76$, 1997.
[11] T. Ellis, A. Abbood, and B. Brillault, "Ellipse Detection and Matching with Uncertainty," Image and Vision Computings, vol. 10, no. 5, pp. 271276, 1992 .

[12] M. S. Nixon, "Circle Extraction via Least Squares and the Kalman Filter," in Proceedings of the 5th International Conference on Computer Analysis of Images and Patterns, 1993.

[13] N. Chernov and C. Lesort, "Least Squares Fitting of Circles," Journal of Mathematical Imaging and Vision, vol. 23, no. 3, pp. 239-252, 2005.

[14] A. Al-Sharadqah and N. Chernov, "Error Analysis for Circle Fitting Algorithms," Electronic Journal of Statistics, vol. 3, pp. 886-911, 2009.

[15] N. N. Chan, "On Circular Functional Relationships," Journal of the Royal Statistical Society. Series $B$ (Methodological), vol. 27, no. 1, pp. 45-56, 1965.

[16] K. Kanatani, Statistical Optimization for Geometric Computation: Theory and Practice. New York, NY, USA: Elsevier Science Inc., 1996.

[17] R. Kan, "From Moments of Sum to Moments of Product," Journal of Multivariate Analysis, vol. 99, no. 3, pp. 542-554, March 2008. 